\title{
Impact of the symmetry energy on nuclear pasta phases and crust-core transition in neutron stars
}

\author{
S. S. Bao and H. Shen* \\ School of Physics, Nankai University, Tianjin 300071, China
}

\begin{abstract}
We study the impact of the symmetry energy on properties of nuclear pasta phases and crustcore transition in neutron stars. We perform a self-consistent Thomas-Fermi calculation employing the relativistic mean-field model. The properties of pasta phases presented in the inner crust of neutron stars are investigated and the crust-core transition is examined. It is found that the slope of the symmetry energy plays an important role in determining the pasta phase structure and the crust-core transition. The correlation between the symmetry energy slope and the crust-core transition density obtained in the Thomas-Fermi approximation is consistent with that predicted by the liquid-drop model.
\end{abstract}

PACS numbers: 26.60.-c, 26.60.Gj, 21.65.Cd

Keywords: Symmetry energy, Pasta phase, Crust-core transition

*Electronic address: shennankai@gmail.com 


\section{INTRODUCTION}

Neutron stars are great laboratories for the study of asymmetric nuclear matter over a wide range of density. With increasing depth in the neutron star, the matter density may rise from the subnuclear region to several times normal nuclear matter density [1, 2]. In general, a neutron star consists of an outer crust of nuclei in a gas of electrons, an inner crust of nuclei in a gas of neutrons and electrons, and a liquid core of uniform dense matter [2 4]. The inner crust of neutron stars has drawn much attention due to its complex phase structure and significant role in astrophysical observations [ $[5]$ ] $]$. In the inner crust, spherical nuclei may become unstable as the density increases toward the crustcore transition, and the stable nuclear shape is likely to change from droplet to rod, slab, tube, and bubble, known as nuclear pasta phases [6-10]. The crust-core transition occurs at the density where the energy density of the homogeneous matter becomes lower than that of the pasta phase. During the last decades, the properties of pasta phases have been investigated by using various methods, such as the liquid-drop model [6, 10, 11] and the Thomas-Fermi approximation [8, 9, 12, 13]. In Ref. [12], the density region of nonspherical nuclei was evaluated by using a parametrized Thomas-Fermi approximation, which was found to be sensitive to the density dependence of the nuclear symmetry energy. In Ref. [8], a self-consistent Thomas-Fermi approximation was used to calculate properties of the inner crust including pasta phases, and it was found that the symmetry energy and its slope could have significant impacts on the pasta phase structure and crust-core transition. In our previous work [10], the effects of the symmetry energy on pasta phase properties and crust-core transition were investigated by employing the coexisting phases method based on a liquid-drop model, and the correlation between the symmetry energy slope and the crust-core transition was obtained and analyzed. It is noticeable that the symmetry energy and its slope could play an important role in determining the pasta phase structure and crust-core transition in neutron stars.

In recent years, the nuclear symmetry energy and its density dependence have received great interest due to their importance for understanding many phenomena in nuclear physics

and astrophysics [14 17]. The value of the symmetry energy $E_{\text {sym }}$ at saturation density is constrained by experiments to be about $30 \pm 4 \mathrm{MeV}$, while its slope $L$ at saturation density is still very uncertain and may vary from about 20 to $115 \mathrm{MeV}$ [18]. It has been 
found that various properties of neutron stars, such as the crust structure, the crust-core transition, and the star radius, are sensitive to the symmetry energy $E_{\text {sym }}$ and its slope $L$ [8, 12, 19, 20]. In Ref. [19], the impact of the symmetry energy on the crust-core transition was examined using various effective Skyrme and relativistic approaches, in which the crustcore transition density obtained from the dynamical and thermodynamical methods showed a clear decrease with increasing $L$. The correlation between the crust-core transition density and the symmetry energy slope $L$ has been extensively studied in the literature using various methods [8, 10, 12, 19]. It is shown that the resulting transition density depends on the method and effective nuclear interaction used in the calculation. It is important to make further investigations in order to determine a clear correlation between the symmetry energy slope $L$ and the crust-core transition.

The main purpose of this article is to investigate the impact of the symmetry energy on pasta phase properties and explore the correlation between the symmetry energy slope $L$ and the crust-core transition. We perform a self-consistent Thomas-Fermi calculation employing the relativistic mean-field (RMF) model [21, 22] for nuclear interactions. In the Thomas-Fermi approximation, the surface effect and nucleon distributions are treated self-consistently, rather than by assuming a sharp interface as in the coexisting phases method [10]. In our most recent study [23], we made a detailed comparison between the Thomas-Fermi (TF) approximation and the coexisting phases (CP) method with only the droplet configuration. It is interesting to compare their difference in pasta phases including all configurations as mentioned above. For the nuclear interaction, we adopt the RMF model with two different parametrizations, TM1 [24] and IUFSU [25], both of which are known to be successful in describing the ground-state properties of finite nuclei, including unstable ones. In the RMF approach, nucleons interact via the exchange of scalar and vector mesons, and the model parameters are generally fitted to nuclear matter saturation properties or ground-state properties of finite nuclei. The TM1 parametrization includes nonlinear terms for both $\sigma$ and $\omega$ mesons, while an additional $\omega$ - $\rho$ coupling term is added in the IUFSU parametrization. It is well known that the $\omega$ - $\rho$ coupling term plays a crucial role in modifying the density dependence of the symmetry energy and affecting the neutron star properties [16, 20, 25 27]. In order to evaluate the impact of the symmetry energy slope $L$ on pasta phase properties and crust-core transition, we employ two sets of generated models based on the TM1 and IUFSU parametrizations as given in Ref. [23]. The model parameters 
were determined by simultaneously adjusting $g_{\rho}$ and $\Lambda_{\mathrm{v}}$ so as to achieve a given $L$ at saturation density $n_{0}$ while keeping $E_{\text {sym }}$ fixed at a density of $0.11 \mathrm{fm}^{-3}$. We note that the fixed density in Ref. [10] was chosen to be the saturation density, namely, $n_{\mathrm{fix}}=n_{0}$, whereas $n_{\mathrm{fix}}$ $=0.11 \mathrm{fm}^{-3}$ was used in Ref. [23]. It has been shown in Ref. [23] that the choice of the fixed density $n_{\mathrm{fix}}=0.11 \mathrm{fm}^{-3}$ could produce very similar binding energies for finite nuclei within one set of generated models. Furthermore, all models in each set have the same isoscalar saturation properties and fixed symmetry energy at $n_{\text {fix }}=0.11 \mathrm{fm}^{-3}$, but they have different symmetry energy slope $L$. By using the set of models with different $L$, it is possible to study the impact of $L$ on pasta phase properties and explore the correlation between $L$ and the crust-core transition.

This article is organized as follows. In Sec. III, we briefly describe the RMF model and the self-consistent TF approximation used in this study. In Sec. III, we present the numerical results and examine the impact of the symmetry energy on pasta phase properties, while the correlation between the symmetry energy slope $L$ and the crust-core transition is discussed. Section IV is devoted to the conclusions.

\section{FORMALISM}

The inner crust of neutron stars is studied within the TF approximation by employing the RMF model for nuclear interactions. In the RMF model [21, 22], nucleons interact through the exchange of various mesons. The mesons considered here are the isoscalar-scalar meson $\sigma$, the isoscalar-vector meson $\omega$, and the isovector-vector meson $\rho$. For a system consisting of protons, neutrons, and electrons, the Lagrangian density reads

$$
\begin{aligned}
\mathcal{L}_{\mathrm{RMF}}= & \sum_{i=p, n} \bar{\psi}_{i}\left\{i \gamma_{\mu} \partial^{\mu}-\left(M+g_{\sigma} \sigma\right)-\gamma_{\mu}\left[g_{\omega} \omega^{\mu}+\frac{g_{\rho}}{2} \tau_{a} \rho^{a \mu}+\frac{e}{2}\left(1+\tau_{3}\right) A^{\mu}\right]\right\} \psi_{i} \\
& +\bar{\psi}_{e}\left[i \gamma_{\mu} \partial^{\mu}-m_{e}+e \gamma_{\mu} A^{\mu}\right] \psi_{e} \\
& +\frac{1}{2} \partial_{\mu} \sigma \partial^{\mu} \sigma-\frac{1}{2} m_{\sigma}^{2} \sigma^{2}-\frac{1}{3} g_{2} \sigma^{3}-\frac{1}{4} g_{3} \sigma^{4} \\
& -\frac{1}{4} W_{\mu \nu} W^{\mu \nu}+\frac{1}{2} m_{\omega}^{2} \omega_{\mu} \omega^{\mu}+\frac{1}{4} c_{3}\left(\omega_{\mu} \omega^{\mu}\right)^{2} \\
& -\frac{1}{4} R_{\mu \nu}^{a} R^{a \mu \nu}+\frac{1}{2} m_{\rho}^{2} \rho_{\mu}^{a} \rho^{a \mu}+\Lambda_{\mathrm{v}}\left(g_{\omega}^{2} \omega_{\mu} \omega^{\mu}\right)\left(g_{\rho}^{2} \rho_{\mu}^{a} \rho^{a \mu}\right)-\frac{1}{4} F_{\mu \nu} F^{\mu \nu},
\end{aligned}
$$

where $W^{\mu \nu}, R^{a \mu \nu}$, and $F^{\mu \nu}$ are the antisymmetric field tensors corresponding to $\omega^{\mu}, \rho^{a \mu}$, and $A^{\mu}$, respectively. In the RMF approach, the meson fields are treated as classical fields, 
and the field operators are replaced by their expectation values. For a static system, the nonvanishing expectation values are $\sigma=\langle\sigma\rangle, \omega=\left\langle\omega^{0}\right\rangle, \rho=\left\langle\rho^{30}\right\rangle$, and $A=\left\langle A^{0}\right\rangle$. The equations of motion for these mean fields derived from the Lagrangian density (11) have the following form:

$$
\begin{aligned}
& -\nabla^{2} \sigma+m_{\sigma}^{2} \sigma+g_{2} \sigma^{2}+g_{3} \sigma^{3}=-g_{\sigma}\left(n_{p}^{s}+n_{n}^{s}\right) \\
& -\nabla^{2} \omega+m_{\omega}^{2} \omega+c_{3} \omega^{3}+2 \Lambda_{\mathrm{v}} g_{\omega}^{2} g_{\rho}^{2} \rho^{2} \omega=g_{\omega}\left(n_{p}+n_{n}\right), \\
& -\nabla^{2} \rho+m_{\rho}^{2} \rho+2 \Lambda_{\mathrm{v}} g_{\omega}^{2} g_{\rho}^{2} \omega^{2} \rho=\frac{g_{\rho}}{2}\left(n_{p}-n_{n}\right) \\
& -\nabla^{2} A=e\left(n_{p}-n_{e}\right)
\end{aligned}
$$

where $n_{i}^{s}$ and $n_{i}$ denote, respectively, the scalar and number densities of species $i$. The equations of motion for nucleons give the standard relations between the densities and chemical potentials,

$$
\begin{aligned}
& \mu_{p}=\sqrt{\left(k_{F}^{p}\right)^{2}+M^{* 2}}+g_{\omega} \omega+\frac{g_{\rho}}{2} \rho+e A, \\
& \mu_{n}=\sqrt{\left(k_{F}^{n}\right)^{2}+M^{* 2}}+g_{\omega} \omega-\frac{g_{\rho}}{2} \rho,
\end{aligned}
$$

where $M^{*}=M+g_{\sigma} \sigma$ is the effective nucleon mass, and $k_{F}^{i}$ is the Fermi momentum of species $i$, which is related to the number density by $n_{i}=\left(k_{F}^{i}\right)^{3} / 3 \pi^{2}$.

The matter in the inner crust of neutron stars contains protons, neutrons, and electrons under the conditions of $\beta$ equilibrium and charge neutrality. We employ the Wigner-Seitz cell approximation to describe the inner crust, in which the equilibrium state is determined by minimization of the total energy density at zero temperature. The stable cell shape may change from droplet to rod, slab, tube, and bubble as the density increases. For simplicity, we assume the electron density is uniform throughout the Wigner-Seitz cell, since the electron screening effect is known to be negligible at subnuclear densities [28]. Furthermore, we also neglect the correction caused by the Coulomb interaction with charged particles in other cells, which is negligibly small in most cases [29, 30]. In the TF approximation, the total energy per cell is calculated from

$$
E_{\text {cell }}=\int_{\text {cell }} \varepsilon_{\text {rmf }}(\mathbf{r}) d \mathbf{r}+\varepsilon_{e} V_{\text {cell }},
$$

where $\varepsilon_{e}$ is the electron kinetic energy density, and $\varepsilon_{\text {rmf }}(\mathbf{r})$ is the local energy density at 
position $\mathbf{r}$, which is given in the RMF model by

$$
\begin{aligned}
\varepsilon_{\mathrm{rmf}}(\mathbf{r})= & \sum_{i=p, n} \frac{1}{\pi^{2}} \int_{0}^{k_{F}^{i}} d k k^{2} \sqrt{k^{2}+M^{* 2}} \\
& +\frac{1}{2}(\nabla \sigma)^{2}+\frac{1}{2} m_{\sigma}^{2} \sigma^{2}+\frac{1}{3} g_{2} \sigma^{3}+\frac{1}{4} g_{3} \sigma^{4} \\
& -\frac{1}{2}(\nabla \omega)^{2}-\frac{1}{2} m_{\omega}^{2} \omega^{2}-\frac{1}{4} c_{3} \omega^{4}+g_{\omega} \omega\left(n_{p}+n_{n}\right) \\
& -\frac{1}{2}(\nabla \rho)^{2}-\frac{1}{2} m_{\rho}^{2} \rho^{2}-\Lambda_{\mathrm{v}} g_{\omega}^{2} g_{\rho}^{2} \omega^{2} \rho^{2}+\frac{g_{\rho}}{2} \rho\left(n_{p}-n_{n}\right) \\
& -\frac{1}{2}(\nabla A)^{2}+e A\left(n_{p}-n_{e}\right) .
\end{aligned}
$$

Here, we consider different pasta configurations, including the droplet, rod, slab, tube, and bubble. The volume of the Wigner-Seitz cell for different configurations can be written as

$$
V_{\text {cell }}= \begin{cases}\frac{4}{3} \pi r_{\mathrm{ws}}^{3} & (\text { droplet and bubble }) \\ l \pi r_{\mathrm{ws}}^{2} & (\text { rod and tube }) \\ 2 r_{\mathrm{ws}} l^{2} & (\text { slab })\end{cases}
$$

where $r_{\mathrm{ws}}$ is the radius of a spherical cell for the droplet and bubble configurations, while the rod and tube have cylindrical shapes with radius $r_{\mathrm{ws}}$ and length $l$, and the slab has width $l$ and thickness $2 r_{\text {ws }}$. We note that the choices of the length for a cylindrical shape and the width for a slab are somewhat arbitrary [8], which would not affect the resulting energy density of the system.

At a given average baryon density $n_{b}$, we minimize the total energy density with respect to the cell size $r_{\mathrm{ws}}$ for each pasta configuration, and then we compare the energy densities between different configurations in order to determine the most stable shape that has the lowest energy density. Furthermore, the energy density of the corresponding homogeneous phase at the same $n_{b}$ is also computed, and the crust-core transition occurs at the density where the energy density of the homogeneous phase becomes lower than that of the pasta phase. In order to calculate the total energy per cell given by Eq. (8) at fixed $r_{\mathrm{ws}}$ and $n_{b}$, we solve the coupled Eqs. (2)-(5) under the constraints of $\beta$ equilibrium, charge neutrality, and baryon number conservation, which have the following form:

$$
\begin{aligned}
\mu_{n} & =\mu_{p}+\mu_{e} \\
N_{e} & =N_{p}=\int_{\text {cell }} n_{p}(\mathbf{r}) d \mathbf{r}, \\
n_{b} V_{\text {cell }} & =\int_{\text {cell }}\left[n_{p}(\mathbf{r})+n_{n}(\mathbf{r})\right] d \mathbf{r} .
\end{aligned}
$$


In practice, we start with an initial guess for the mean fields $\sigma(\mathbf{r}), \omega(\mathbf{r}), \rho(\mathbf{r})$, and $A(\mathbf{r})$, then determine the chemical potentials $\mu_{n}, \mu_{p}$, and $\mu_{e}$ by the constraints (11)-(13). Once the chemical potentials are obtained, it is easy to calculate various densities and solve Eqs. (2)(5) to get new mean fields. This procedure should be iterated until convergence is achieved.

\section{RESULTS AND DISCUSSION}

In this section, we present numerical results for the inner crust of neutron stars, and we discuss the impact of the symmetry energy on pasta phase properties and crust-core transition. The results obtained from the self-consistent TF calculation are compared with those obtained using the CP method [10]. For the effective nuclear interaction, we consider two successful RMF models, TM1 [24] and IUFSU [25]. The parameter sets and saturation properties of these two models are given in Tables \and II, respectively. In order to clarify the correlation between the symmetry energy slope $L$ and the crust-core transition, we employ two sets of generated models based on the TM1 and IUFSU parametrizations as given in Ref. [23]. It is noticeable that all models in each set have the same isoscalar saturation properties and fixed symmetry energy $E_{\text {sym }}$ at a density of $0.11 \mathrm{fm}^{-3}$ but have different symmetry energy slope $L$. These models have been generated by simultaneously adjusting $g_{\rho}$ and $\Lambda_{\mathrm{v}}$ so as to achieve a given $L$ at saturation density $n_{0}$ while keeping $E_{\text {sym }}$ fixed at a density of $0.11 \mathrm{fm}^{-3}$ as described in Ref. [23]. The parameters, $g_{\rho}$ and $\Lambda_{\mathrm{v}}$, generated from the TM1 and IUFSU models for different $L$ are given in Tables III and [IV, respectively. In Fig. 1, we plot the symmetry energy $E_{\text {sym }}$ as a function of the baryon density $n_{b}$ for the two sets of models generated from TM1 (upper panel) and IUFSU (lower panel). One can see that all models in each set have the same $E_{\text {sym }}$ at a density of $0.11 \mathrm{fm}^{-3}$, but they have different values of $E_{\text {sym }}$ at lower and higher densities due to the difference in the slope $L$. It is obvious that a smaller $L$ corresponds to a larger (smaller) $E_{\text {sym }}$ at lower (higher) densities. It will be shown below that the behavior of $E_{\text {sym }}$ plays a crucial role in determining the pasta phase structure and the crust-core transition.

We first present the phase diagram for the inner crust of neutron stars and discuss the influence of the symmetry energy slope $L$ on the pasta phase structure. In Fig. 2, the density ranges of various pasta phases obtained from the self-consistent TF calculation are displayed for the two sets of generated models, IUFSU (left panel) and TM1 (right panel). It is found 
that only the droplet configuration can occur before the crust-core transition for $L \geq 80$ $\mathrm{MeV}$, whereas the pasta phase structure may change from droplet to rod, slab, tube, and bubble for smaller values of $L$ (e.g., $L=50 \mathrm{MeV}$ ). As one can see from Fig. 2, the onset density of nonspherical nuclei, i.e., the transition density from droplet to rod, significantly decreases with decreasing $L$ in the low- $L$ region $(L \leq 70 \mathrm{MeV})$. This behavior is consistent with that reported in Ref. [12]. It can be understood from a fission-like instability of spherical nuclei predicted in the liquid-drop model [12, 31], in which a spherical liquid drop becomes unstable to quadrupolar deformations when the volume fraction of the liquid drop reaches the fission-instability criterion, $u=\left(r_{d} / r_{\mathrm{ws}}\right)^{3}=1 / 8$, with $r_{d}$ and $r_{\mathrm{ws}}$ being, respectively, the radii of the droplet and the Wigner-Seitz cell. From Fig. 4 below, we can see that a smaller $L$ corresponds to a larger value of $r_{d} / r_{\text {ws }}$ in the droplet phase at low densities, and, therefore, the model with a smaller $L$ results in an earlier onset of nonspherical nuclei, as shown in Fig. 2. On the other hand, the onset density of homogeneous matter significantly decreases with increasing $L$, which has also been observed in earlier studies [8, 10, 12, 17]. This trend may be understood from the energy-density curvature of pure neutron matter. According to the analysis in the liquid-drop model [17], the energy-density curvature of pure neutron matter at saturation density, $C_{\mathrm{NM}}\left(n_{0}\right)$, is approximately proportional to $L$, and the inhomogeneous phase occurs in the spinodal region of asymmetric nuclear matter where the energy density has a negative curvature. A rough analysis implies that the larger $C_{\mathrm{NM}}\left(n_{0}\right)$ is, the farther away a spinodal border of $\beta$-equilibrium matter deviates from the saturation density $n_{0}$. Therefore, the model with a larger $L$ leads to a smaller onset density of homogeneous matter, as shown in Fig. 2[see also Fig. 9(a)]. We conclude that a smaller value of $L$ results in an earlier onset of nonspherical nuclei and a later transition to homogeneous matter. Hence, the model with a smaller $L$ predicts a more complex phase structure and a larger density range of pasta phases in neutron star crusts.

The phase diagram obtained in the present TF calculation is very similar to that obtained using the CP method [10]. However, the bubble configuration could not appear even with the lowest $L$ by using the CP method [10]. In order to make a detailed comparison of the pasta phase structure between the TF and CP calculations, we present in Table $\mathrm{V}$ the onset densities of pasta phases for the original IUFSU $(L=47.2 \mathrm{MeV})$ and TM1 $(L=110.8 \mathrm{MeV})$ models. It is shown that only the droplet configuration appears in the case of TM1 for both $\mathrm{TF}$ and $\mathrm{CP}$, which is due to its very large value of $L$. On the other hand, the original IUFSU 
model predicts that almost all pasta phases can occur before the crust-core transition due to its small value of $L$. From Table $\mathrm{V}$, we can see that there are visible differences in the onset densities between TF and CP. This is mainly caused by the different treatments of surface and Coulomb energies [23], which play an essential role in determining the phase shape. It is found that the onset density of homogeneous matter, i.e., the crust-core transition density, obtained in the TF approximation is slightly higher than that obtained using the CP method. This is because the configuration space of TF is much larger than that of CP, and, therefore, a lower energy density can be achieved in the minimization procedure of the TF calculation, which leads to a higher crust-core transition density. In Fig. 3, we plot the energy per nucleon of the pasta phase relative to that of homogeneous matter, $\Delta E$, as a function of the average baryon density, $n_{b}$, obtained from the $\mathrm{TF}$ and $\mathrm{CP}$ calculations using the original IUFSU model. One can see that there are significant differences in $\Delta E$ between TF and CP at lower densities, whereas the differences become much smaller in the pasta phase region. The comparison between TF and CP at low density with the droplet configuration has been extensively discussed in our previous work [23], and it was found that the simple CP method could not describe the nonuniform matter around the neutron drip density due to its energy being higher than that of homogeneous matter. From Fig. 3, it is seen that $\Delta E$ of the CP calculation becomes positive at $n_{b}<0.003 \mathrm{fm}^{-3}$, which is consistent with the onset density of the droplet phase given in Table V. On the other hand, the crust-core transition occurs at relatively high density, beyond which $\Delta E$ becomes positive. Although the differences in $\Delta E$ between $\mathrm{TF}$ and $\mathrm{CP}$ calculations at higher densities are rather small (on the order of a few $\mathrm{keV}$ ), it may lead to visible differences in the onset densities as shown in Table V. We note that the differences in the energy per nucleon between different pasta shapes are of the order of $0.1-1 \mathrm{keV}$, which has also been reported in Refs. [11, 29]. Therefore, the pasta phase structure is very sensitive to the method and nuclear interaction used in the calculation.

In the present study, we focus on the correlation between the symmetry energy slope $L$ and pasta phase properties. In Fig. 4, the size of the Wigner-Seitz cell, $r_{\text {ws }}$, and that of the inner part (nucleus or hole), $r_{d}$, which are obtained from the TF calculation with two extreme values of $L$ in the TM1 (upper panel) and IUFSU (lower panel) sets, are displayed as a function of $n_{b}$. In the TF approximation, there is no distinct boundary between the liquid phase and the gas phase, so we prefer to define the size of the inner part, $r_{d}$, by density 
fluctuations, similar to that of Refs. [7, 28], as

$$
r_{d}= \begin{cases}r_{\mathrm{ws}}\left(\frac{\left\langle n_{p}\right\rangle^{2}}{\left\langle n_{p}^{2}\right\rangle}\right)^{1 / D} & (\text { droplet, rod, and slab) } \\ r_{\mathrm{ws}}\left(1-\frac{\left\langle n_{p}\right\rangle^{2}}{\left\langle n_{p}^{2}\right\rangle}\right)^{1 / D} & \text { (tube and bubble) }\end{cases}
$$

with $D=1,2,3$ being the geometrical dimension of the system. The average values, $\left\langle n_{p}\right\rangle$ and $\left\langle n_{p}^{2}\right\rangle$, are calculated over the cell volume $V_{\text {cell }}$ given by Eq. (10). From Fig. 4, one can see that the model with the smallest $L$ yields a rather complex structure of the inner crust, whereas only the droplet phase appears in the case with the largest $L$. It is found that $r_{\text {ws }}$ and $r_{d}$ show significant jumps at the transition points between different pasta shapes, which indicates that the transition is first order. One can see that $r_{\text {ws }}$ decreases with $n_{b}$ at lower densities, while it rapidly increases before the crust-core transition in the case of small $L$. A similar behavior of $r_{\mathrm{ws}}$ was also observed in Ref. [8].

It is of interest to compare the results between the TF and CP methods. In Fig. 5(a), $r_{\text {ws }}$ and $r_{d}$ obtained in the TF approximation are compared with those obtained by the CP method using the original IUFSU model. It is found that both $r_{\mathrm{ws}}$ and $r_{d}$ from the CP method are somewhat smaller than those from the TF method. This can be explained by the behaviors of the electron chemical potential $\mu_{e}$ shown in Fig. 5(b) and the average proton fraction $Y_{p}$ shown in Fig. 55(c). As discussed in Ref. [23], the CP method could yield larger $\mu_{e}$ and $Y_{p}$ in comparison to those of TF [see Figs. 5(b) and 5)(c)]. This is because the surface and Coulomb energies are treated perturbatively in the CP method, whereas they are included self-consistently in the minimization of the TF method. Due to larger $\mu_{e}$ and $Y_{p}$, the CP method would give rise to smaller $r_{\mathrm{ws}}$ as shown in Fig. 5(a), which could be explained by their correlation in the liquid-drop model [23]. From Figs. 5(b) and 5)(c), one can see that there are small jumps at $n_{b} \sim 0.079 \mathrm{fm}^{-3}$ in the TF case, which are caused by the transition from slab to tube. It is obvious that trends in the differences between CP and TF are very similar for all pasta phases, and they are consistent with those obtained in the droplet phase [23].

To examine the influence of $L$ on properties of the inner crust, we perform self-consistent TF calculations using the two sets of generated models based on the TM1 and IUFSU parametrizations. In Fig. 6, we present the following quantities: (a) the average proton fraction $Y_{p}$; (b) neutron densities of the liquid phase and the gas phase, $n_{n, L}$ and $n_{n, G}$, at the center or boundary of the cell; and (c) the proton density of the liquid phase, $n_{p, L}$, at 
the center or boundary of the cell. As one can see from Fig. 6(a), $Y_{p}$ decreases rapidly with increasing $n_{b}$ at lower densities and shows a significant $L$ dependence at higher densities. It is found that a smaller $L$ corresponds to a larger $Y_{p}$ at a fixed $n_{b}$. This trend is related to the density dependence of $E_{\mathrm{sym}}$ shown in Fig. 1. Since $E_{\mathrm{sym}}$ has been fixed at $n_{b}=0.11 \mathrm{fm}^{-3}$, a smaller $L$ in one set of generated models corresponds to a larger $E_{\text {sym }}$ at lower densities $\left(n_{b}<0.11 \mathrm{fm}^{-3}\right)$. It is well known that a larger $E_{\text {sym }}$ favors a higher $Y_{p}$ in homogeneous $\beta$-equilibrium matter. Therefore, a smaller $L$ results in a larger $Y_{p}$ at the density close to the transition to homogeneous matter. The correlation between $L$ and $Y_{p}$ is consistent with those reported in Refs. [8, 12]. A clear $L$ dependence is also observed in Fig. 6(b), in which a smaller $L$ corresponds to larger $n_{n, L}$ and smaller $n_{n, G}$. The $L$ dependence of $n_{n, L}$ and $n_{n, G}$ can be explained by the density dependence of $E_{\text {sym }}$ as discussed above. It is shown in Fig. 1 that a smaller $L$ corresponds to larger $E_{\text {sym }}$ at $n_{b}<0.11 \mathrm{fm}^{-3}$ and smaller $E_{\text {sym }}$ at $n_{b}>0.11 \mathrm{fm}^{-3}$. Therefore, the model with a smaller $L$ favors more neutrons in the liquid phase and fewer neutrons in the gas phase, which results in larger $n_{n, L}$ and smaller $n_{n, G}$, as shown in Fig. 6(b). We note that the behaviors of $n_{n, L}$ and $n_{n, G}$ obtained in the present study are consistent with those reported in Refs. [8, 12]. On the other hand, the behavior of $n_{p, L}$ is somewhat complicated, as shown in Fig. 6(c). The model with a smaller $L$ results in a more rapid decrease of $n_{p, L}$ at $0.01<n_{b}<0.05 \mathrm{fm}^{-3}$. This trend may be related to the $\omega-\rho$ coupling term and behaviors of chemical potentials as discussed in Ref. [23]. In Fig. 7, we display chemical potentials of electrons, $\mu_{e}$, neutrons, $\mu_{n}$, and protons, $\mu_{p}$, as a function of $n_{b}$ obtained in the TF approximation for the two sets of generated models with several values of $L$. The chemical potentials are intensive quantities that play an important role in the TF calculation. With increasing $n_{b}$, both $\mu_{e}$ and $\mu_{n}$ increase monotonically in all cases of $L$, whereas $\mu_{p}$ decreases. For the $L$ dependence of chemical potentials at a fixed $n_{b}$, it is found that the model with a smaller $L$ generally results in larger $\mu_{e}$ and $\mu_{n}$, as well as smaller $\mu_{p}$. This can be explained by the contribution from the $\rho$ meson in the RMF model. According to Eqs. (66) and (7), the value of $g_{\rho} \rho$ plays an essential role in determining the chemical potentials $\mu_{p}, \mu_{n}$, and $\mu_{e}=\mu_{n}-\mu_{p}$. One can see from Tables [II] and [V] that the model with a smaller $L$ has relatively larger $g_{\rho}$ and $\Lambda_{\mathrm{v}}$, which yields a larger value of $g_{\rho} \rho$. Therefore, the model with a smaller $L$ leads to larger $\mu_{e}$ and $\mu_{n}$, as well as smaller $\mu_{p}$. By comparing results between TM1 and IUFSU, we find that the two sets of generated models have similar $L$ dependence for all properties mentioned above. These results are also 
consistent with those reported in Refs. [8, 10, 12, 23].

It is interesting to see how the density profile evolves through various pasta phases as the density increases. In Fig. 8, the density distributions of neutrons and protons inside the Wigner-Seitz cell are plotted at several values of $n_{b}$. The calculations are performed with two extreme values of $L$ in the set of IUFSU $(L=47.2 \mathrm{MeV}$ and $L=110 \mathrm{MeV})$. As shown in Fig. 2, the original IUFSU model $(L=47.2 \mathrm{MeV})$ predicts that all pasta phases would occur before the crust-core transition, whereas only the droplet configuration appears in the case of $L=110 \mathrm{MeV}$. In Fig. 8, from top to bottom, we show the results of droplet, rod, slab, tube, and bubble, respectively. In the top panel at $n_{b}=0.04 \mathrm{fm}^{-3}$, the results for a droplet with $L=47.2 \mathrm{MeV}$ (thick lines) are compared to those with $L=110 \mathrm{MeV}$ (thin lines). It is seen that the droplet size obtained with $L=47.2 \mathrm{MeV}$ is larger than that with $L=110 \mathrm{MeV}$, and the nucleon distributions in the case of $L=110 \mathrm{MeV}$ are more diffuse than those of $L=47.2 \mathrm{MeV}$. Moreover, as compared to the case of $L=110$ $\mathrm{MeV}$, the original IUFSU model with $L=47.2 \mathrm{MeV}$ yields lower neutron gas density at the boundary $\left(n_{n, G}\right)$ and higher neutron density at the center of the cell $\left(n_{n, L}\right)$, which are consistent with those shown in Fig. 6(b). In the second panel at $n_{b}=0.06 \mathrm{fm}^{-3}$, the original IUFSU model predicts a rod phase, while the model with $L=110 \mathrm{MeV}$ has undergone the crust-core transition. The transition from slab to tube occurs at $n_{b} \sim 0.079 \mathrm{fm}^{-3}$ in the original IUFSU model, and, therefore, sudden changes in the density distributions are observed by comparing the third and fourth panels; these cause visible jumps in chemical potentials and $Y_{p}$ as shown above. As one can see, from top to bottom, the distributions of neutrons and protons become more diffuse with increasing $n_{b}$, and the neutron density difference between the liquid phase and the gas phase becomes smaller and smaller. The crust-core transition occurs at $n_{b} \sim 0.092 \mathrm{fm}^{-3}$ in the original IUFSU model.

Finally, we discuss the correlation between the symmetry energy slope $L$ and the crustcore transition. In Fig. 9, we display the crust-core transition density $n_{b, t}$ and the proton fraction and the pressure at the transition point, $Y_{p, t}$ and $P_{t}$, as a function of $L$ obtained from the self-consistent TF calculation using the two sets of generated models based on the TM1 and IUFSU parametrizations. As one can see from Fig. 9)(a), there is a clear correlation between $L$ and $n_{b, t}$; namely, $n_{b, t}$ decreases monotonically with increasing $L$. This correlation is consistent with those reported in Refs. [8, 10, 12, 19]. Compared to the results obtained using the CP method [10], the transition densities obtained from the present TF 
calculation are slightly higher, which may be related to the differences in configuration space and treatments of surface and Coulomb energies. From Fig. 9(b), it is seen that $Y_{p, t}$ decreases significantly with increasing $L$. This trend is very similar to that observed in Refs. [10, 19]. It can be understood from the density dependence of $E_{\text {sym }}$ shown in Fig. 1. As mentioned above, a smaller $L$ in one set of generated models corresponds to a larger $E_{\text {sym }}$ at $n_{b}<0.11$ $\mathrm{fm}^{-3}$, and a larger $E_{\text {sym }}$ favors a higher $Y_{p}$ in homogeneous $\beta$-equilibrium matter. Therefore, a smaller $L$ results in a larger $Y_{p, t}$ as illustrated in Fig. 9(b). The $L$ dependence of the transition pressure $P_{t}$ is nonmonotonic, as shown in Fig. 9(c). It is found that $P_{t}$ decreases with increasing $L$ in the large- $L$ region $(L>60 \mathrm{MeV})$, whereas the opposite behavior is observed for $L<60 \mathrm{MeV}$. A similar behavior was also observed in Ref. [10]. The nontrivial $L$ dependence of $P_{t}$ is due to a competing effect, as discussed in Refs. [10, 17]. For neutronrich matter at fixed density and proton fraction, the pressure would increase with increasing $L$. However, the decrease of $n_{b, t}$ shown in Fig. 9(a) leads to a decrease of the pressure with increasing $L$. As a result, $P_{t}$ depends on $L$ nonmonotonically, as illustrated in Fig. 9(c).

\section{CONCLUSIONS}

In this study, we have investigated the impact of the symmetry energy on pasta phase properties within the self-consistent TF approximation. It has been found that the symmetry energy slope $L$ plays an important role in determining the pasta phase structure and the crust-core transition. In order to clarify the influence of $L$, we have employed two sets of generated models based on the TM1 and IUFSU parametrizations that have the same isoscalar saturation properties and fixed symmetry energy at the density $n_{b}=0.11 \mathrm{fm}^{-3}$ but have different symmetry energy slope $L$. It has been observed that the model with a smaller $L$ predicts an earlier onset of nonspherical nuclei and a later transition to homogeneous matter. For example, the original IUFSU model with $L=47.2 \mathrm{MeV}$ predicts that the transition from droplet to rod occurs at $n_{b} \sim 0.049 \mathrm{fm}^{-3}$, then the pasta phases of slab, tube, and bubble appear one by one, and finally the transition to homogeneous matter occurs at $n_{b} \sim 0.092 \mathrm{fm}^{-3}$. In contrast, the original TM1 model with $L=110.8 \mathrm{MeV}$ predicts that only the droplet configuration appears at low density, and the transition from droplet to homogeneous matter occurs at $n_{b} \sim 0.062 \mathrm{fm}^{-3}$. Therefore, it can be concluded that the model with a smaller $L$ results in a more complex phase structure and a larger density range 
of pasta phases for neutron star crusts. In addition, some properties of the inner crust such as the proton fraction, chemical potentials, and density profiles have also been found to be correlated to the symmetry energy slope $L$, which could be partly explained by the density dependence of the symmetry energy. We have compared the results of the present TF calculation with those obtained using the CP method. It has been found that, although there are quantitative differences between these two methods, the qualitative behaviors of the inner crust are very similar to each other, and they are consistent with those reported in earlier studies [8, 12].

The correlation between the symmetry energy slope $L$ and the crust-core transition has been examined in the TF approximation using the two sets of models generated from the TM1 and IUFSU parametrizations. It has been found that the crust-core transition density $n_{b, t}$ decreases monotonically with increasing $L$. This correlation is consistent with those reported in Refs. [8, 10, 12, 19]. Also, the proton fraction at the transition point $Y_{p, t}$ decreases significantly with increasing $L$, whereas the transition pressure $P_{t}$ shows a nontrivial dependence on $L$. These correlations obtained from the present TF calculation are consistent with those obtained using the CP method [10]. In the TF approximation, nuclear shell and pairing effects have been neglected. It is of interest to further consider neutron paring and superfluidity in the inner crust of neutron stars.

\section{Acknowledgment}

This work was supported in part by the National Natural Science Foundation of China (Grant No. 11375089). 
[1] J. M. Lattimer and M. Prakash, Science 304, 536 (2004).

[2] N. Chamel and P. Haensel, Living Rev. Relativ. 11, 10 (2008).

[3] H. Heiselberg and M. Hjorth-Jensen, Phys. Rep. 328, 237 (2000).

[4] F. Weber, Prog. Part. Nucl. Phys. 54, 193 (2005).

[5] A. W. Steiner, Phys. Rev. C 77, 035805 (2008).

[6] D. G. Ravenhall, C. J. Pethick, and J. R. Wilson, Phys. Rev. Lett. 50, 2066 (1983).

[7] M. Okamoto, T. Maruyama, K. Yabana, and T. Tatsumi, Phys. Rev. C 88, 025801 (2013).

[8] F. Grill, C. Providência, and S. S. Avancini, Phys. Rev. C 85, 055808 (2012).

[9] S. S. Avancini, D. P. Menezes, M. D. Alloy, J. R. Marinelli, M. M. W. Moraes, and C. Providência, Phys. Rev. C 78, 015802 (2008).

[10] S. S. Bao and H. Shen, Phys. Rev. C 89, 045807 (2014).

[11] G. Watanabe, K. Iida, and K. Sato, Nucl. Phys. A676, 455 (2000).

[12] K. Oyamatsu and K. Iida, Phys. Rev. C 75, 015801 (2007).

[13] S. S. Avancini, S. Chiacchiera, D. P. Menezes, and C. Providência, Phys. Rev. C 82, 055807 (2010); 85, 059904(E) (2012).

[14] J. M. Lattimer and M. Prakash, Phys. Rep. 442, 109 (2007).

[15] B. A. Li, L. W. Chen, and C. M. Ko, Phys. Rep. 464, 113 (2008).

[16] C. J. Horowitz and J. Piekarewicz, Phys. Rev. Lett. 86, 5647 (2001).

[17] C. Ducoin, J. Margueron, and C. Providência, Europhys. Lett. 91, 32001 (2010).

[18] Z. Zhang and L. W. Chen, Phys. Lett. B726, 234 (2013).

[19] C. Ducoin, J. Margueron, C. Providência, and I. Vidana, Phys. Rev. C 83, 045810 (2011).

[20] R. Cavagnoli, D. P. Menezes, and C. Providência, Phys. Rev. C 84, 065810 (2011).

[21] B. D. Serot and J. D. Walecka, Adv. Nucl. Phys. 16, 1 (1986).

[22] J. Meng, H. Toki, S. G. Zhou, S. Q. Zhang, W. H. Long, and L. S. Geng, Prog. Part. Nucl. Phys. 57, 470 (2006).

[23] S. S. Bao, J. N. Hu, Z. W. Zhang, and H. Shen, Phys. Rev. C 90, 045802 (2014).

[24] Y. Sugahara and H. Toki, Nucl. Phys. A 579, 557 (1994).

[25] F. J. Fattoyev, C. J. Horowitz, J. Piekarewicz, and G. Shen, Phys. Rev. C 82, 055803 (2010).

[26] J. Carriere, C. J. Horowitz, and J. Piekarewicz, Astrophys. J. 593, 463 (2003). 
[27] C. Providência and A. Rabhi, Phys. Rev. C 87, 055801 (2013).

[28] T. Maruyama, T. Tatsumi, D. N. Voskresensky, T. Tanigawa, and S. Chiba, Phys. Rev. C 72 , $015802(2005)$.

[29] K. Oyamatsu, Nucl. Phys. A 561, 431 (1993).

[30] H. Shen, H. Toki, K. Oyamatsu, and K. Sumiyoshi, Astrophys. J. Suppl. 197, 20 (2011).

[31] K. Iida, G. Watanabe, and K. Sato, Prog. Theor. Phys. Suppl. 146, 514 (2002). 
TABLE I: Parameter sets used in this work. The masses are given in MeV.

\begin{tabular}{cccccccccccc}
\hline \hline Model & $M$ & $m_{\sigma}$ & $m_{\omega}$ & $m_{\rho}$ & $g_{\sigma}$ & $g_{\omega}$ & $g_{\rho}$ & $g_{2}\left(\mathrm{fm}^{-1}\right)$ & $g_{3}$ & $c_{3}$ & $\Lambda_{\mathrm{v}}$ \\
\hline TM1 & 938.0 & 511.198 & 783.0 & 770.0 & 10.0289 & 12.6139 & 9.2644 & -7.2325 & 0.6183 & 71.3075 & 0.000 \\
IUFSU & 939.0 & 491.500 & 782.5 & 763.0 & 9.9713 & 13.0321 & 13.5900 & -8.4929 & 0.4877 & 144.2195 & 0.046 \\
\hline \hline
\end{tabular}

TABLE II: Saturation properties of nuclear matter for the TM1 and IUFSU models. The quantities $E_{0}, K, E_{\text {sym }}$, and $L$ are, respectively, the energy per nucleon, incompressibility coefficient, symmetry energy, and symmetry energy slope at saturation density $n_{0}$.

\begin{tabular}{lccccc}
\hline \hline Model & $n_{0}\left(\mathrm{fm}^{-3}\right)$ & $E_{0}(\mathrm{MeV})$ & $K(\mathrm{MeV})$ & $E_{\mathrm{sym}}(\mathrm{MeV})$ & $L(\mathrm{MeV})$ \\
\hline TM1 & 0.145 & -16.3 & 281.0 & 36.9 & 110.8 \\
IUFSU & 0.155 & -16.4 & 231.0 & 31.3 & 47.2 \\
\hline \hline
\end{tabular}

TABLE III: Parameters, $g_{\rho}$ and $\Lambda_{\mathrm{v}}$, generated from the TM1 model for different slope $L$ at saturation density $n_{0}$ with fixed symmetry energy $E_{\text {sym }}=28.05 \mathrm{MeV}$ at a density of $0.11 \mathrm{fm}^{-3}$. The last line shows the symmetry energy at saturation density, $E_{\mathrm{sym}}\left(n_{0}\right)$. The original TM1 model has $L=110.8 \mathrm{MeV}$.

\begin{tabular}{lcccccccc}
\hline \hline$L(\mathrm{MeV})$ & 40.0 & 50.0 & 60.0 & 70.0 & 80.0 & 90.0 & 100.0 & 110.8 \\
\hline$g_{\rho}$ & 13.9714 & 12.2413 & 11.2610 & 10.6142 & 10.1484 & 9.7933 & 9.5114 & 9.2644 \\
$\Lambda_{\mathrm{v}}$ & 0.0429 & 0.0327 & 0.0248 & 0.0182 & 0.0128 & 0.0080 & 0.0039 & 0.0000 \\
$E_{\text {sym }}\left(n_{0}\right)(\mathrm{MeV})$ & 31.38 & 32.39 & 33.29 & 34.11 & 34.86 & 35.56 & 36.22 & 36.89 \\
\hline \hline
\end{tabular}


TABLE IV: Parameters, $g_{\rho}$ and $\Lambda_{\mathrm{v}}$, generated from the IUFSU model for different slope $L$ at saturation density $n_{0}$ with fixed symmetry energy $E_{\text {sym }}=26.78 \mathrm{MeV}$ at a density of $0.11 \mathrm{fm}^{-3}$. The last line shows the symmetry energy at saturation density, $E_{\mathrm{sym}}\left(n_{0}\right)$. The original IUFSU model has $L=47.2 \mathrm{MeV}$.

\begin{tabular}{lcccccccc}
\hline \hline$L(\mathrm{MeV})$ & 47.2 & 50.0 & 60.0 & 70.0 & 80.0 & 90.0 & 100.0 & 110.0 \\
\hline$g_{\rho}$ & 13.5900 & 12.8202 & 11.1893 & 10.3150 & 9.7537 & 9.3559 & 9.0558 & 8.8192 \\
$\Lambda_{\mathrm{v}}$ & 0.0460 & 0.0420 & 0.0305 & 0.0220 & 0.0153 & 0.0098 & 0.0051 & 0.0011 \\
$E_{\text {sym }}\left(n_{0}\right)(\mathrm{MeV})$ & 31.30 & 31.68 & 32.89 & 33.94 & 34.88 & 35.74 & 36.53 & 37.27 \\
\hline \hline
\end{tabular}

TABLE V: Comparison of the onset densities of pasta phases and homogeneous matter (hom.) between the TF and CP methods for the original IUFSU and TM1 models.

\begin{tabular}{lccccccccc}
\hline \hline Model & \multirow{2}{*}{ Method } & \multicolumn{5}{c}{ Onset density $\left(\mathrm{fm}^{-3}\right)$} \\
\cline { 3 - 8 } & & droplet & rod & slab & tube & bubble hom. \\
\hline IUFSU & TF & 0.000 & 0.049 & 0.063 & 0.079 & 0.085 & 0.092 \\
IUFSU & CP & 0.003 & 0.055 & 0.070 & 0.088 & - & 0.089 \\
TM1 & TF & 0.000 & - & - & - & - & 0.062 \\
TM1 & CP & 0.001 & - & - & - & - & 0.058 \\
\hline \hline
\end{tabular}




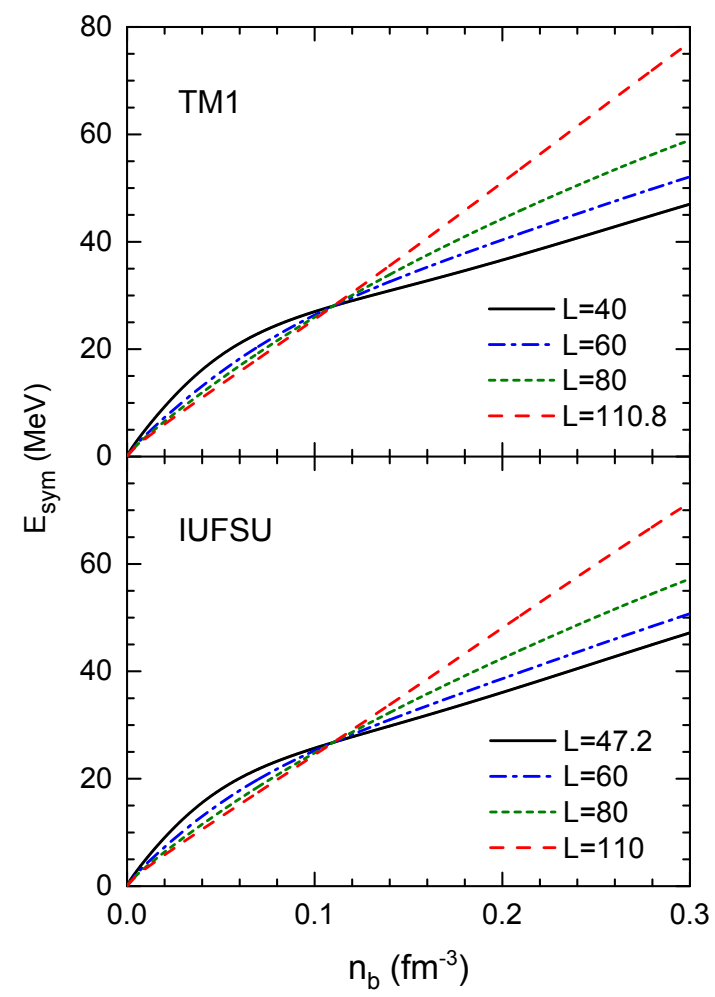

FIG. 1: (Color online) Symmetry energy $E_{\text {sym }}$ as a function of the baryon density $n_{b}$ for modified versions of TM1 (upper panel) and IUFSU (lower panel) with several values of $L$ at saturation density. The symmetry energy is fixed at a density of $0.11 \mathrm{fm}^{-3}$.

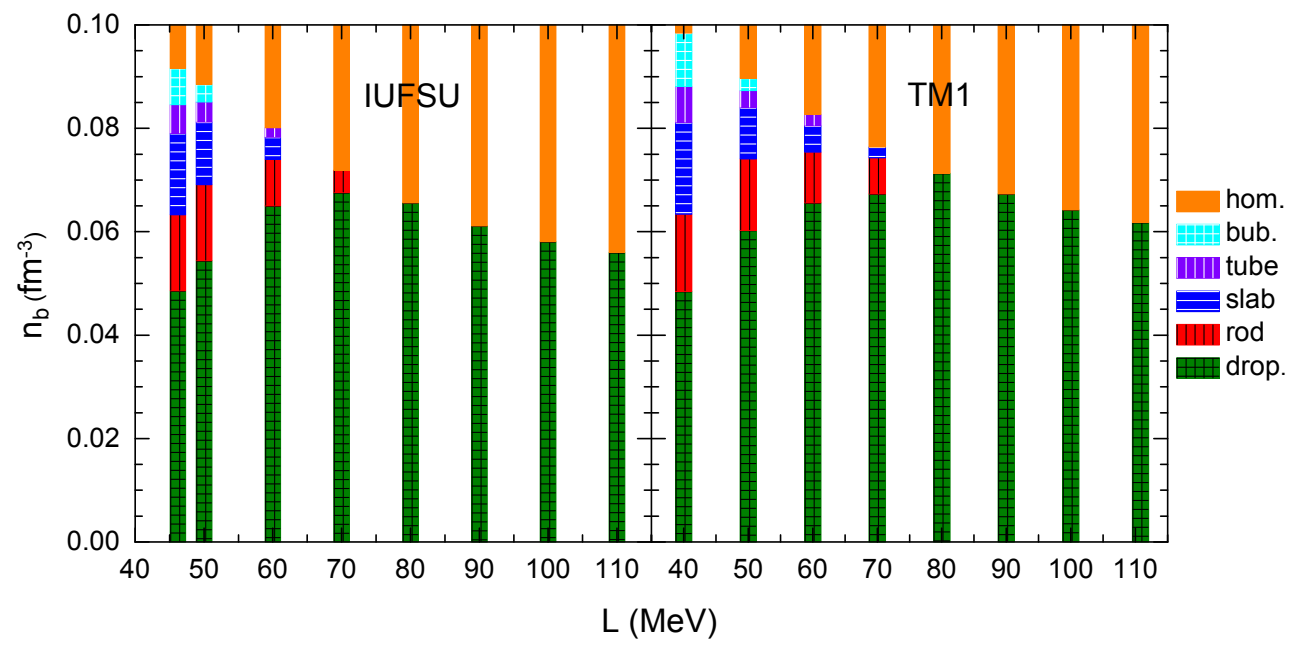

FIG. 2: (Color online) Phase diagrams for the two sets of models generated from IUFSU (left panel) and TM1 (right panel). Different colors represent droplet, rod, slab, tube, bubble, and homogeneous phases as indicated in the legend. 


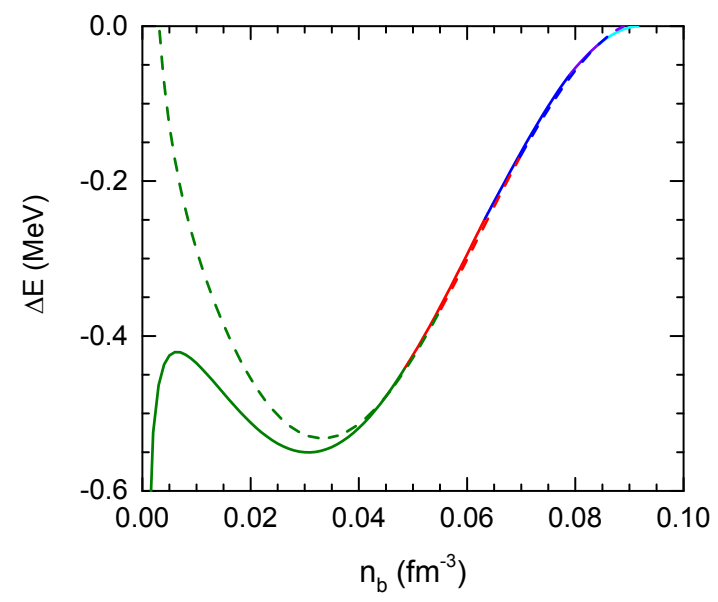

FIG. 3: (Color online) Energy per nucleon of the pasta phase relative to that of homogeneous matter, $\Delta E$, as a function of $n_{b}$ obtained from the TF (solid lines) and CP (dashed lines) calculations using the original IUFSU model. Different colors represent different pasta phases as shown in Fig. 2, 


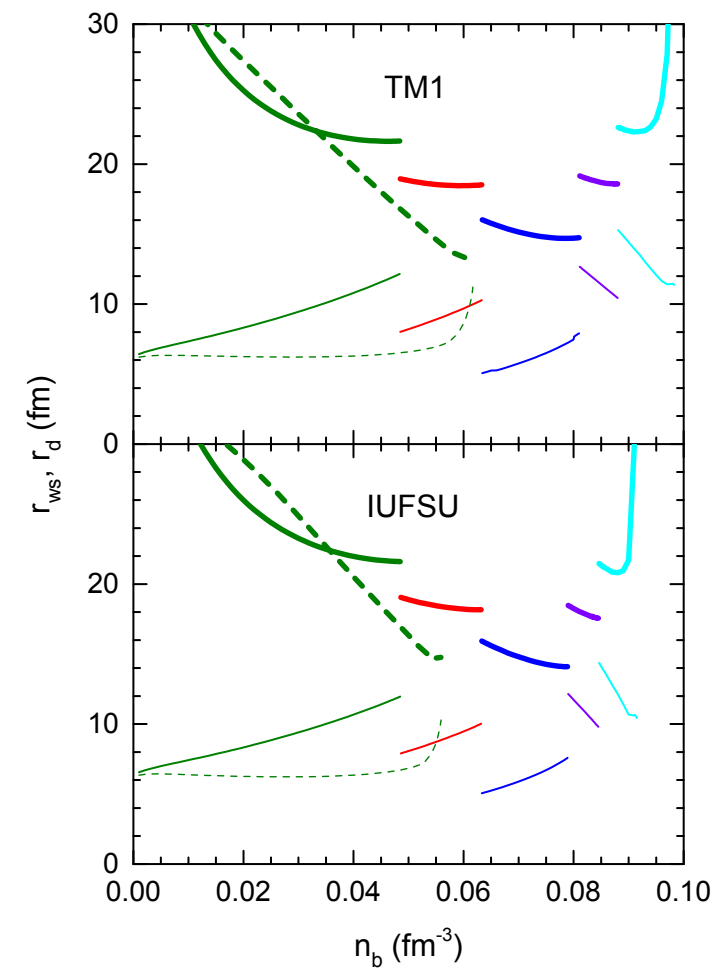

FIG. 4: (Color online) Size of the Wigner-Seitz cell, $r_{\mathrm{ws}}$ (thick lines), and that of the inner part, $r_{d}$ (thin lines), as a function of $n_{b}$ obtained in the TF approximation. The results calculated with the smallest $L$ in TM1 $(L=40 \mathrm{MeV})$ and IUFSU $(L=47.2 \mathrm{MeV})$ are shown by solid lines, where the pasta phase structure changes from droplet (green) to rod (red), slab (blue), tube (violet), and bubble (cyan) as the density increases. For comparison, the results with the largest $L$ in TM1 $(L=110.8 \mathrm{MeV})$ and IUFSU $(L=110 \mathrm{MeV})$ are shown by green-dashed lines, where only the droplet configuration appears before the crust-core transition. 

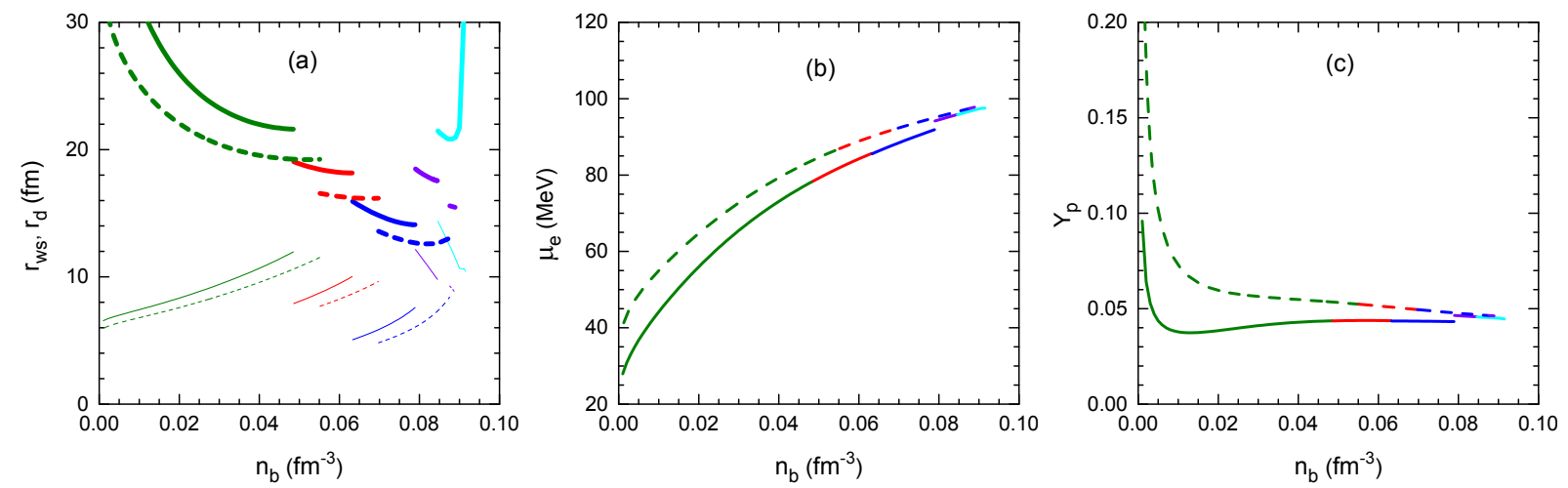

FIG. 5: (Color online) Comparison of equilibrium properties of the inner crust between the TF (solid lines) and CP (dashed lines) methods using the original IUFSU model. The size of the Wigner-Seitz cell, $r_{\mathrm{ws}}$ (thick lines), and that of the inner part, $r_{d}$ (thin lines) (a), the electron chemical potential $\mu_{e}(\mathrm{~b})$, and the average proton fraction $Y_{p}(\mathrm{c})$ are plotted as a function of $n_{b}$. The different colors represent different pasta phases.
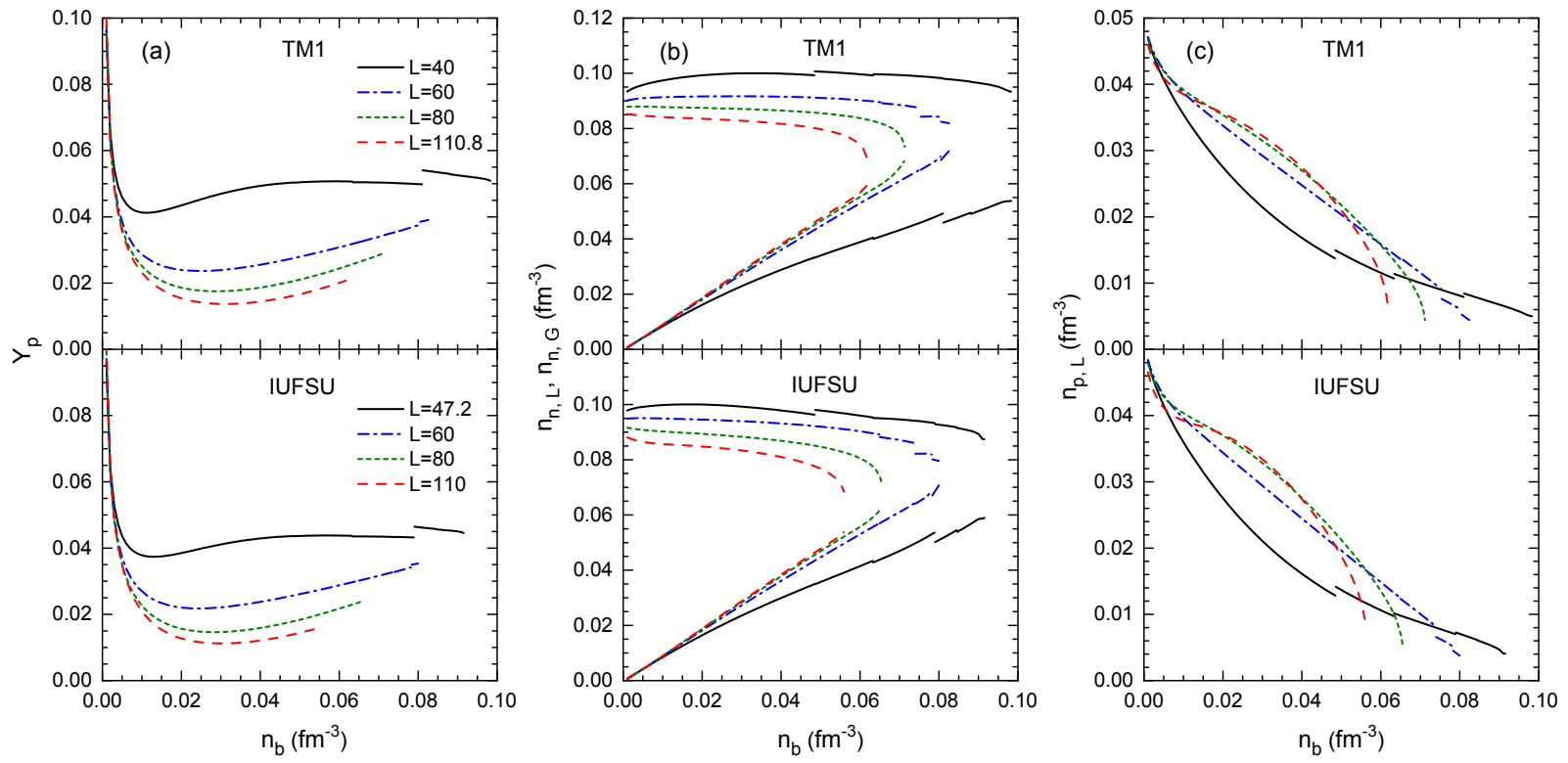

FIG. 6: (Color online) Equilibrium properties of the Wigner-Seitz cell obtained in the TF approximation for modified versions of TM1 (upper panel) and IUFSU (lower panel) with several values of $L$. The average proton fraction $Y_{p}(\mathrm{a})$, the neutron densities of the liquid phase and the gas phase, $n_{n, L}$ and $n_{n, G}$, at the center or boundary of the cell (b), and the proton density of the liquid phase, $n_{p, L}$, at the center or boundary of the cell (c) are plotted as a function of $n_{b}$. Small jumps are observed at the transition between different pasta shapes. 

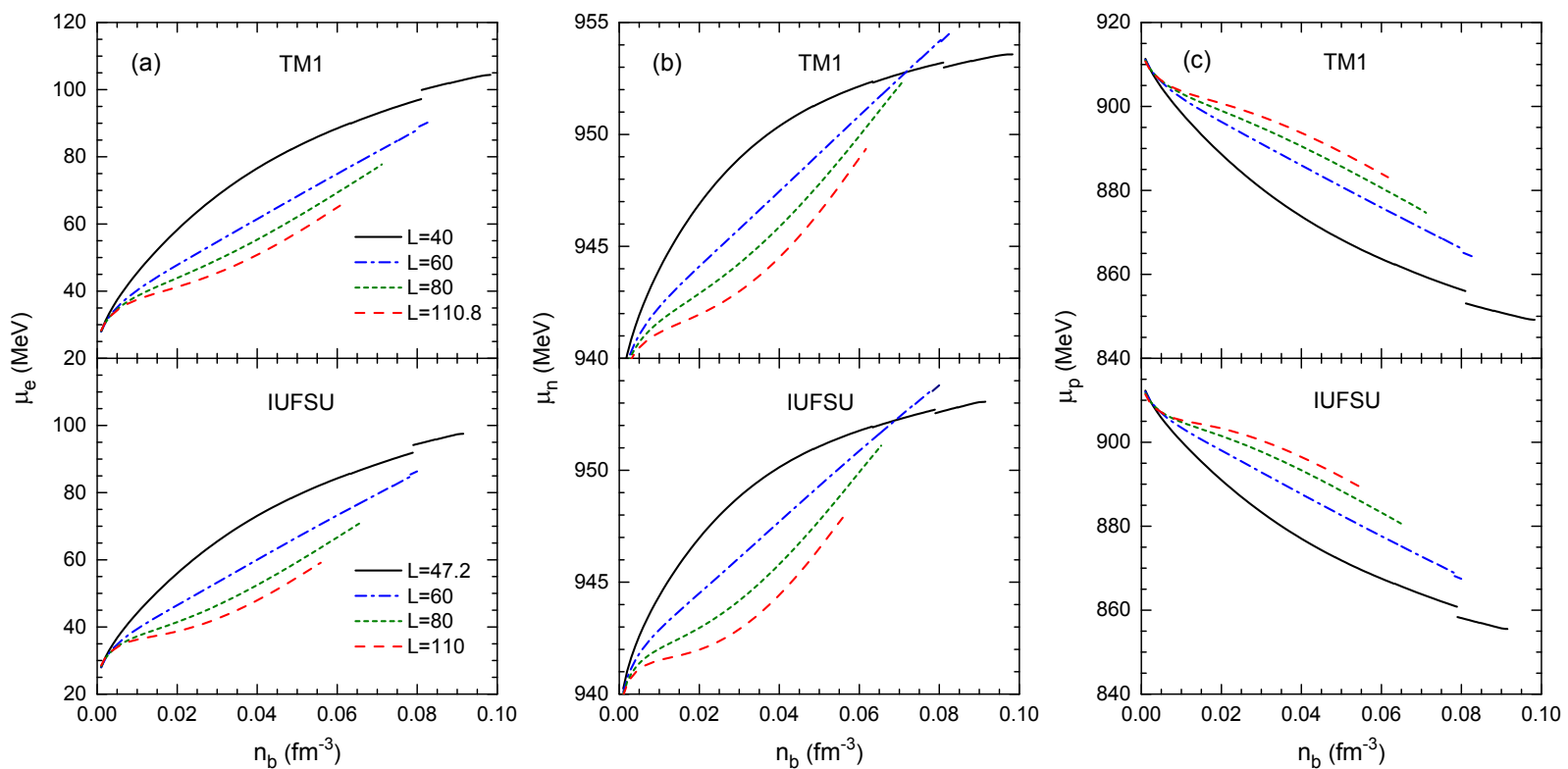

FIG. 7: (Color online) Same as Fig. 6, but for chemical potentials of electrons $\mu_{e}$ (a), neutrons $\mu_{n}$ (b), and protons $\mu_{p}(\mathrm{c})$. 


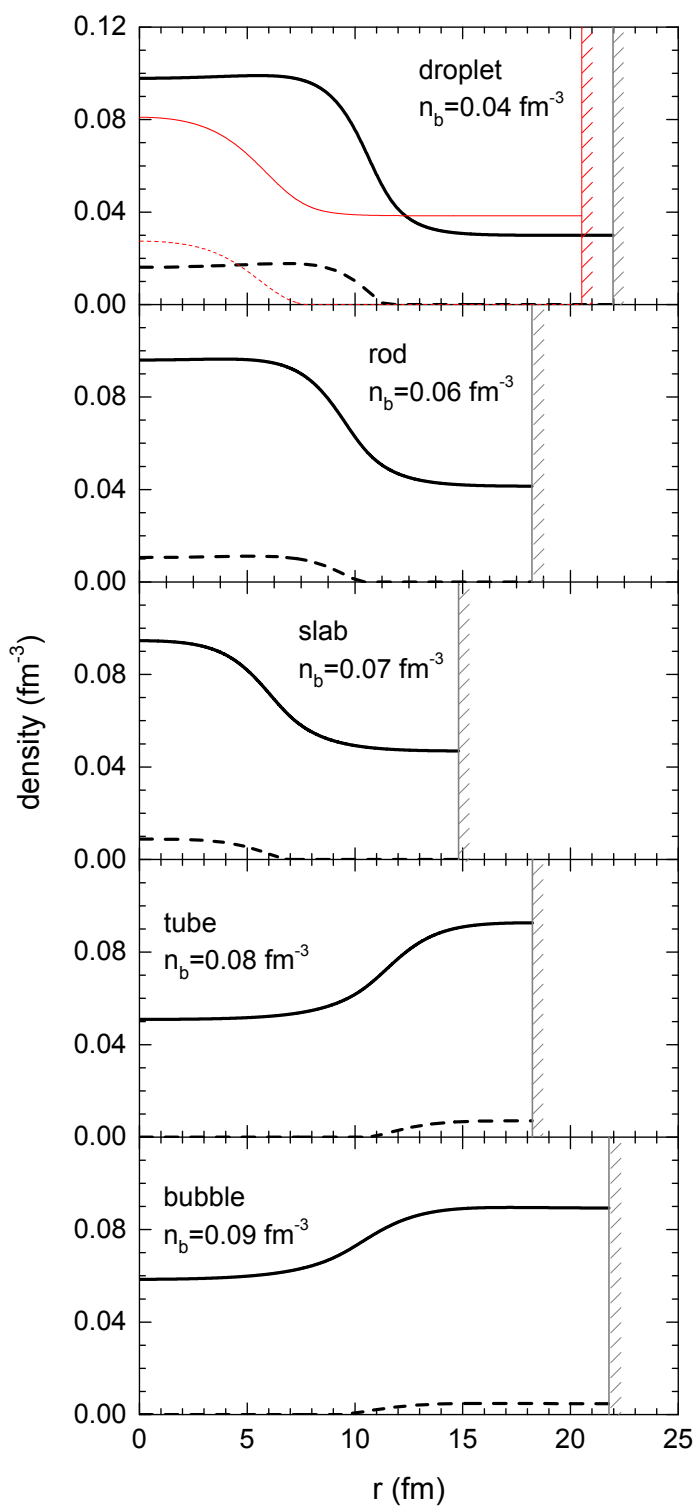

FIG. 8: (Color online) Density distributions of neutrons (solid lines) and protons (dashed lines) in the Winger-Seitz cell at $n_{b}=0.04,0.06,0.07,0.08$, and $0.09 \mathrm{fm}^{-3}$ (top to bottom) obtained in the TF approximation. The results with the original IUFSU model $(L=47.2 \mathrm{MeV})$ are shown by thick black lines. For comparison, the results with the largest $L$ in the set of IUFSU $(L=110$ $\mathrm{MeV}$ ) are shown by thin red lines in the top panel. The cell boundary is indicated by the hatching. 

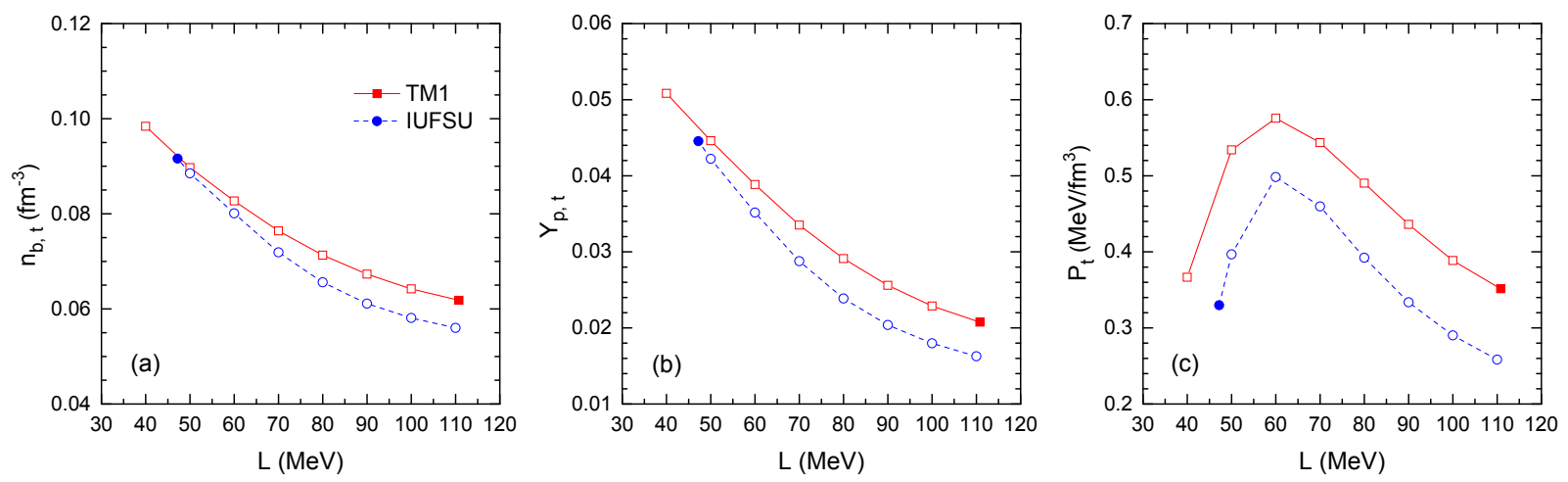

FIG. 9: (Color online) Crust-core transition density $n_{b, t}(\mathrm{a})$, proton fraction at the transition point, $Y_{p, t}(\mathrm{~b})$, and pressure at the transition point, $P_{t}(\mathrm{c})$, as a function of $L$ obtained in the TF approximation using the two sets of models generated from TM1 (red-solid line with squares) and IUFSU (blue-dashed line with circles). The results obtained with the original TM1 and IUFSU models are indicated by the filled square and circle, respectively. 